\title{
Adverse childhood experiences and adult self-harm in a female forensic population
}

\author{
Rachel Holden, ${ }^{1,2, \uparrow}$ (1) Imogen Stables, ${ }^{2, \uparrow}$ (1) Penelope Brown, ${ }^{1,2}$ Maria Fotiadou ${ }^{1}$
}

BJPsych Bulletin (2022) 46, 148-152, doi:10.1192/bjb.2021.34

${ }^{1}$ South London and Maudsley NHS Foundation Trust, UK; ${ }^{2}$ King's College London, UK

Correspondence to Dr Rachel Holden (rachel.holden@kcl.ac.uk)

First received 27 Jul 2020, final revision 14 Feb 2021, accepted 30 Mar 2021

(c) The Author(s), 2021. Published by Cambridge University Press on behalf of the Royal College of Psychiatrists. This is an Open Access article, distributed under the terms of the Creative Commons Attribution licence (http:// creativecommons.org/licenses/by/4. O/), which permits unrestricted re-use, distribution, and reproduction in any medium, provided the original work is properly cited.

\begin{abstract}
Aims and method This study aimed to investigate the prevalence of adverse childhood experiences (ACEs) among patients in a female forensic psychiatric in-patient medium-secure unit, and to analyse the link between ACEs, adulthood self-harm and associated comorbidities and risk factors. The study used a cross-sectional design, with data gathered from the anonymised electronic health records of patients.
\end{abstract}

Results It was found that there was a high prevalence of both ACEs and self-harm among this patient group, and that there was a relationship between the two; those with more ACEs were more likely to have self-harmed during adulthood. Of the individual ACE categories, it was also demonstrated that emotional abuse had a significant association with adulthood self-harm.

Clinical implications In medium-secure settings for women, implementation of trauma-informed care will be beneficial because of the high number of those with mental disorders who have experienced adversity during their childhood.

Keywords Trauma; forensic mental health services; in-patient treatment; self-harm; adverse childhood experiences.

\begin{abstract}
Adverse childhood experiences (ACEs) are stressful or traumatic life events that occur before 18 years of age. ${ }^{1,2}$ Having a history of ACEs is not uncommon: in a national household survey of adults residing in England, $47 \%$ of participants reported at least one $\mathrm{ACE} .^{3}$ Research on ACEs has demonstrated links between ACEs and self-harm. ${ }^{4}$ In a female prison population, all types of childhood abuse and neglect were more prevalent in those who self-harmed compared with those who did not, with significant associations between adulthood self-harm and both childhood emotional and sexual abuse. ${ }^{5}$ ACEs have a cumulative effect on health outcomes, with higher numbers of ACEs predicting more adverse health outcomes. ${ }^{6}$

This study occurred on Spring Ward, the female ward of River House Medium Secure Unit (MSU) in South London and Maudsley NHS Foundation Trust. Since opening in April 2008, the ward has offered a multidisciplinary biopsychosocial approach to support women toward recovery and reintegration into the community. ${ }^{7}$ In 2017 , following the success of implementing the Healing Trauma programme (a gender-specific intervention for trauma victims), ${ }^{8}$ a trauma-informed care approach was introduced. Implementation involved team consultation, presentation and a training session on Stephanie Covington's Becoming Trauma-Informed Programme. ${ }^{9}$
\end{abstract}

\footnotetext{
${ }^{\dagger}$ Joint first authors.
}

This study was conducted as part of a service evaluation to determine the prevalence of ACEs in a female MSU cohort and to explore the relationship between ACEs and adulthood selfharm. Links between ACEs and adulthood self-harming behaviours, violence and comorbidities (such as personality disorder) will be explored, which will enable us to better understand the present and future needs of patients.

\begin{abstract}
Aims
Our first aim was to establish the prevalence of ACEs in patients in a female MSU, using a structured ACE questionnaire. Second, we aimed to establish the prevalence of adulthood self-harm, personality disorder, alcohol and drug misuse, and history of violence among female in-patients in the MSU. And finally, we aimed to explore the relationship between ACEs and adulthood self-harm.
\end{abstract}

\section{Method}

\section{Design and procedure}

The main study utilised a cross-sectional design, using medical records. The researchers conducted a thorough review of each patient's electronic medical records. Using previously recorded information, the amount of ACEs were calculated by the researcher, based upon the trauma history recorded in the patients records. 


\section{Participants}

All patients who were admitted to or were receiving continuing treatment on the ward between April 2008 and July 2019 were considered eligible for participation in this study. Criteria for admission to the ward include being over 18 years of age, committing an offence or having a significant history of violence. All patients are sectioned under the Mental Health Act 1983 (MHA) throughout their admission. The ward can accommodate 15 patients. Because of the relapsing and remitting nature of the mental disorders of many patients, several patients had multiple admissions during the study period; for these patients, data was gathered from medical records documented during their most recent admission.

Initially, 68 participants were included in the sample. Two participants were excluded from the analysis because of insufficient information in their medical records regarding their childhood, taking the final sample to 66 participants. Demographic and clinical information included age on admission, ethnicity, primary diagnosis, MHA section, personality disorder, alcohol and drug misuse, and violence history. Ethnicity was recorded into subsections: White British, Black British, Black African, Black Caribbean, Asian and other. Primary diagnosis was recorded into three categories, according to the ICD-10: schizophrenia spectrum disorders (codes F20-F29), mood [affective] disorders (codes F30-F39) and personality disorders (codes F60F69) ${ }^{10}$ MHA sections of all patients were recorded into categories: forensic (section 37, sections 37 and 41, sections 47 and 49 , and sections 48 and 49) and civil (section 3). ${ }^{11}$

\section{Data collection and analysis}

Exposure to adverse experiences up to 18 years of age was assessed for each participant, following a thorough review of their medical notes; ACE history was summarised by a ten-item version of the Adverse Childhood Experiences Questionnaire. ${ }^{12}$ The ACE questionnaire consists of ten binary (yes/no) questions that assess exposure to emotional, physical and sexual abuse; emotional and physical neglect; and household dysfunction, including domestic violence, substance use and incarceration. Participants' self-harm history since 18 years of age was obtained via medical records, along with comorbid personality disorder, alcohol and drug misuse, and history of violence. Self-harm was coded as a binary variable, with the presence of self-harm being recorded if there was any mention of self-harm or suicide attempt in adulthood mentioned in the medical records. ACEs were extracted from records of patients' trauma history recorded in psychological and psychiatric reports contained in their medical records. Personality disorder presence was defined as a previous diagnosis of any type of personality disorder. Alcohol and drug misuse were defined as any positive history of problematic use of alcohol or drugs. History of violence was defined as any violence history before the event leading to admission. Each of these variables were recorded as dichotomous (yes/no) variables.

Data was analysed with the Statistical Package for Social Sciences (IBM SPSS, version 25 for Mac).

\section{Ethical approval}

Ethical approval was granted by the Research, Outcomes and Service Evaluation Committee, a branch of the Behavioural and Developmental Psychiatry Clinical Academic Group of South London and Maudsley NHS Foundation Trust. The ethical approval included the use of anonymised medical records. This was a service development project using historic clinical records and as such the study was exempt from a need to provide informed consent.

\section{Results}

Patients were aged between 18 and 72 years at admission (Table 1). The sample was primarily Black and minority ethnic, and most patients had a primary diagnosis of schizophrenia, schizotypal and delusional disorders (ICD-10 codes F20-F29). ${ }^{10}$ The majority of the sample had a history of drug misuse. Over a third of the sample had a history of alcohol misuse, and over a quarter had a comorbid personality disorder diagnosis.

\section{Number and prevalence of ACEs}

In the total sample, the mean number of ACEs was 2.89 $( \pm 2.35)$ (Table 2), with ACE number showing a positively skewed distribution. Within the sample, most individuals

Table 1 Demographic and clinical characteristics of the patient sample

\begin{tabular}{|c|c|}
\hline Characteristic & Cases $(N=66)$ \\
\hline Age at admission (years), mean (s.d.) & $38.40( \pm 11.37)$ \\
\hline \multicolumn{2}{|l|}{ Age categories (years), $n(\%)$} \\
\hline$<25$ & $10(15.2)$ \\
\hline $25-34$ & $13(19.7)$ \\
\hline $35-44$ & $26(39.3)$ \\
\hline $45-54$ & $12(18.2)$ \\
\hline$\geq 55$ & $5(7.6)$ \\
\hline \multicolumn{2}{|l|}{ Ethnicity, $n(\%)$} \\
\hline White British & $20(30.3)$ \\
\hline Black British & $13(19.7)$ \\
\hline Black African & $13(19.7)$ \\
\hline Black Caribbean & $10(15.2)$ \\
\hline Asian & $2(3.0)$ \\
\hline Other & $8(12.1)$ \\
\hline \multicolumn{2}{|l|}{ Primary diagnosis, $n(\%)$} \\
\hline $\begin{array}{l}\text { F20-F29, Schizophrenia, schizotypal and } \\
\text { delusional disorders }\end{array}$ & $57(86.4)$ \\
\hline F30-F39, Mood [affective] disorders & $4(6.1)$ \\
\hline $\begin{array}{l}\text { F60-F69, Disorders of adult personality and } \\
\text { behaviour }\end{array}$ & $5(7.6)$ \\
\hline Comorbid personality disorder, $n(\%)$ & $20(30.3)$ \\
\hline Alcohol misuse, $n(\%)$ & $25(37.9)$ \\
\hline Drug misuse, $n(\%)$ & $34(51.5)$ \\
\hline
\end{tabular}




\begin{tabular}{|c|c|}
\hline & Cases, $N=66$ \\
\hline Number of ACEs, mean (s.d.) & $2.89(2.35)$ \\
\hline \multicolumn{2}{|l|}{ Number of ACEs, $n(\%)$} \\
\hline 0 & $12(18.2)$ \\
\hline 1 & $10(15.2)$ \\
\hline 2 & $14(21.2)$ \\
\hline 3 & $5(7.6)$ \\
\hline 4 & $6(9.1)$ \\
\hline 5 & $7(10.6)$ \\
\hline 6 & $7(10.6)$ \\
\hline 7 & $4(6.1)$ \\
\hline 8 & $0(0.0)$ \\
\hline 9 & $1(1.5)$ \\
\hline 10 & $0(0.0)$ \\
\hline \multicolumn{2}{|l|}{$\mathrm{ACE}, n(\%)$} \\
\hline Emotional abuse & $27(40.9)$ \\
\hline Physical abuse & $27(40.9)$ \\
\hline Sexual abuse & $20(30.3)$ \\
\hline Emotional neglect & $26(39.4)$ \\
\hline Physical neglect & $20(30.3)$ \\
\hline Parental separation or divorce & $26(39.4)$ \\
\hline Violence against mother & $8(12.1)$ \\
\hline Household alcohol/drug misuse & $13(19.7)$ \\
\hline Mental illness in household & $21(31.8)$ \\
\hline Incarceration of household member & $2(3.0)$ \\
\hline
\end{tabular}

Within the sample, two was the most common amount of ACEs (56\% of the sample experienced two or more ACEs) and emotional and physical abuse were the most common ACE categories. ACE, adverse childhood experience.

( $n=54,81.9 \%)$ had experienced at least one ACE during childhood; of the total sample, $28.8 \%$ had experienced two to three ACEs and $37.9 \%$ had experienced four or more ACEs. Among the ACE categories, emotional and physical abuse were the most common, and the rarest ACE was incarceration of household members (Table 2).

\section{Number of ACEs and adulthood self-harm}

Adulthood self-harm in the sample was analysed. It was determined that over half of the sample had self-harmed during adulthood ( $n=36,54.5 \%)$. Because of the positive skewed nature of the number of ACEs, we ran a Spearman's correlation to assess the relationship between number of ACEs and adulthood self-harm on the total sample of 66 patients. There was a statistically significant positive correlation between number of ACEs and adulthood self-harm $\left(r_{\mathrm{s}}(64)\right.$ $=0.45, P<0.001)$. Following this, binary logistic regression was performed to ascertain the effect of increasing number of ACEs on the likelihood of adulthood self-harm. The binary logistic regression model was statistically significant $\left(\chi^{2}(1)=\right.$ 15.11, $P<0.005$ ). The model explained $27.4 \%$ (Nagelkerke $R^{2}$ ) of the variance in adulthood self-harm, and correctly classified $75.7 \%$ of cases (specificity $83.3 \%$, sensitivity $69.4 \%$ ). For every one-point increase in number of ACEs, individuals were 1.62 times more likely to self-harm as adults.

\section{ACE categories and adulthood self-harm}

We conducted an analysis to determine whether there was any association between individual ACE categories and adulthood self-harm. The total sample who had self-harmed during adulthood was analysed to determine the prevalence of each type of ACE within this category. In those who had self-harmed during adulthood, emotional abuse was the most common ACE $(n=22,33.3 \%)$, followed by physical abuse $(n=19,28.8 \%)$, emotional neglect $(n=19,28.8 \%)$ and physical neglect $(n=17,25.8 \%)$. Estimates of the odds ratio for each of the ten ACEs ranged from 1.38 (95\% CI $0.47-$ 3.99) for sexual abuse to 8.05 (95\% CI 2.07-31.39) for physical neglect. However, statistically significant associations with adulthood self-harm were only seen for emotional abuse, physical abuse, emotional neglect and physical neglect. These relationships were then further explored by Pearson's $\chi^{2}$-tests and Fisher's exact tests, which confirmed that four categories of ACE had a statistically significant association with adulthood self-harm: emotional abuse $\left(\chi^{2}(1)=13.37, P<0.001\right)$, physical abuse $\left(\chi^{2}(1)=4.62, P=\right.$ $0.032)$, emotional neglect $\left.\left(\chi^{2}(1)=5.94\right), P=0.015\right)$ and physical neglect $(P=0.001$, Fisher's exact test, two-sided).

Multivariate binary logistic regression was conducted to determine whether emotional abuse, physical abuse, emotional neglect and physical neglect maintained their statistically significant association with adulthood self-harm when all four categories were accounted for. In this model, $31.2 \%$ (Nagelkerke $R^{2}$ ) of the variation in adulthood selfharm was explained by these four ACE categories. This model correctly classified $71.2 \%$ of cases (specificity $80.0 \%$, sensitivity $63.9 \%$ ). When accounting for emotional abuse, physical abuse, emotional neglect and physical neglect, only emotional abuse maintained a statistically significant relationship with adulthood self-harm $(P=0.034)$, showing that being emotionally abused increased the likelihood of adulthood self-harm by 7.36 times (Table 3 ).

\section{Discussion}

This study was the first to analyse the prevalence of ACEs, adulthood self-harm and their relationship in a female MSU population in the UK. Multivariate binary logistic regression revealed a statistically significant association between an increasing number of ACEs and increased likelihood of adulthood-self harm. Emotional abuse was shown to have a statistically significant association with adulthood self-harm.

We found a high prevalence of ACE exposure in this female MSU cohort, with over $80 \%$ of individuals experiencing at least one ACE and 56\% experiencing more than two ACEs. The prevalence of ACE exposure among this female MSU group was higher than the $47 \%$ prevalence in the general adult population of the UK. ${ }^{3}$ 
Table 3 Multivariate binary logistic regression to analyse the association between emotional abuse, physical abuse, emotional neglect and physical neglect, and adulthood self-harm

\begin{tabular}{|c|c|c|c|c|c|c|c|c|c|}
\hline & \multirow[b]{2}{*}{ Variables } & \multirow[b]{2}{*}{$B$} & \multirow[b]{2}{*}{ s.e. } & \multirow[b]{2}{*}{ Wald } & \multirow[b]{2}{*}{$\underline{\text { d.f. }}$} & \multirow[b]{2}{*}{$\underline{\text { Significance }}$} & \multirow[b]{2}{*}{$\underline{\operatorname{Exp}(B)}$} & \multicolumn{2}{|c|}{$95 \% \mathrm{Cl}$ for $\operatorname{Exp}(B)$} \\
\hline & & & & & & & & Lower & Upper \\
\hline \multirow[t]{4}{*}{ Step 1} & Emotional abuse & 1.997 & 0.944 & 4.477 & 1 & 0.034 & 7.364 & 1.158 & 46.805 \\
\hline & Physical abuse & -0.507 & 0.905 & 0.314 & 1 & 0.575 & 0.602 & 0.102 & 3.549 \\
\hline & Emotional neglect & -0.965 & 1.116 & 0.748 & 1 & 0.387 & 0.381 & 0.043 & 3.395 \\
\hline & Physical neglect & 1.927 & 1.138 & 2.868 & 1 & 0.090 & 6.868 & 0.739 & 63.859 \\
\hline
\end{tabular}

This multivariate binary logistic regression model included all adverse childhood experience categories that were individually significantly associated with adulthood self-harm. It was run to determine whether statistical significance was maintained when all the categories were accounted for; only emotional abuse remained significantly associated with adulthood self-harm.

Within this population, there was a high prevalence of adulthood self-harm. Adulthood self-harm in our sample was reported by $54.5 \%$, similar to the rates reported by Ribeiro et $\mathrm{al}^{13}$ (whose MSU population overlapped with ours), who found that $46.7 \%$ had a documented history of self-harm before MSU admission. Baker et $\mathrm{al}^{14}$ interviewed female patients in a medium-secure setting and discussed their experiences of self-harm; an overarching theme discussed was that of 'the traumatised individual', suggesting that individuals linking their traumatic experiences to selfharming behaviour is not uncommon. The high prevalence of ACEs amongst the female MSU population indicates that a trauma-informed approach to care in MSU settings for women is crucial. Application of 'universal trauma precautions' is necessary, to ensure that all who have been exposed to ACEs receive care that is not only growthpromoting, but also less likely to cause re-traumatisation than standard care. ${ }^{15}$

We found a statistically significant correlation between an increasing number of ACEs and the likelihood of adulthood self-harm. This is similar to research by Cleare et al, ${ }^{16}$ showing that those with a history of repeat self-harm were significantly more likely to report exposure to multiple ACEs. Moreover, our finding of a statistically significant relationship between emotional abuse and adulthood self-harm supports the research by Howard et al, ${ }^{5}$ who found a statistically significant association between emotional abuse and self-harm in a sample of female prisoners.

It was advantageous to focus on those admitted to a single female MSU over the past 11 years, as there is limited data regarding this population. Use of electronic medical records to obtain data meant minimal information was missing and there was low attrition. In this niche population, we achieved a good sample size; only around $12 \%$ of the 3500 MSU beds in the UK are occupied by women. ${ }^{17}$

The ACE questionnaire is limited as it provides no information regarding the severity, degree, duration, timing or quality of each ACE component, which may differ significantly from person to person. Furthermore, data collected about self-harm behaviours could have been improved by using the Inventory of Statements about Self-Injury questionnaire, ${ }^{18}$ examining the type, frequency, severity and reasons for the behaviour. Further research could focus on collecting more in-depth childhood histories from patients, or using self-harm measures that capture frequency and severity.
Limited research is also available in forensic psychiatric settings, specifically MSUs, and nationwide research into ACEs and self-harm within these units could be beneficial. Furthermore, the neurodevelopmental and psychological mechanisms by which ACEs and self-harm are linked need exploration.

\section{About the authors}

Rachel Holden is a clinical psychologist at South London and Maudsley NHS Foundation Trust, UK, and a Clinical Psychologist Lecturer with the Institute of Psychiatry, Psychology \& Neuroscience at King's College London, UK Imogen Stables is a Medical Student at GKT School of Medical Education, King's College London, UK. Penelope Brown is a Consultant Forensic Psychiatrist at South London and Maudsley NHS Foundation Trust, UK Maria Fotiadou is a Consultant Forensic Psychiatrist South London and Maudsley NHS Foundation Trust, UK

\section{Data availability}

The data that support the findings of this study are available from the corresponding author, R.H., upon reasonable request.

\section{Author contributions}

I.S. was involved in data collection, data analysis and writing of the manuscript. R.H., P.B. and M.F. contributed to writing the manuscript.

\section{Declaration of interest}

None

\section{References}

1 Public Health Scotland. Adverse Childhood Experiences (ACEs). Public Health Scotland, 2019 (http://www.healthscotland.scot/populationgroups/children/adverse-childhood-experiences-aces/overview-of-aces).

2 Public Health Wales. Cymru Well Wales: Adverse Childhood Experiences (ACEs). Public Health Wales, 2018 (http://www.wales.nhs.uk/sitesplus/ 888/page/88524).

3 Bellis MA, Hughes K, Leckenby N, Perkins C, Lowey H. National household survey of adverse childhood experiences and their relationship with resilience to health-harming behaviors in England. BMC Med 2014; 12: 72.

4 Yates TM, Carlson EA, Egeland B. A prospective study of child maltreatment and self-injurious behavior in a community sample. Dev Psychopathol 2008; 20(2): 651-71. 
5 Howard R, Thanos K, Power K, Mahoney A. From childhood trauma to self-harm: an investigation of theoretical pathways among female prisoners. Clin Psychol Psychother 2017; 24(4): 942-51.

6 Hughes K, Bellis M, Hardcastle K, Sethi D, Butchart A, Mikton C, et al. The effect of multiple adverse childhood experiences on health: a systematic review and meta-analysis. Lancet Public Health 2017; 2(8): e356-66.

7 Tully J, Cappai A, Lally J, Fotiadou M. Follow-up study of 6.5 years of admissions to a UK female medium secure forensic psychiatry unit. BJPsych Bulletin 2019; 43(2): 54-7.

8 Covington S, Russo R. Healing Trauma: A Brief Intervention for Women, 1st ed. Hazelden Publishing, 2011.

9 Covington S. Becoming Trauma Informed: A Training for Correctional Professionals, 1st ed. Center for Gender \& Justice, 2012.

10 World Health Organization (WHO). International Statistical Classification of Diseases and Related Health Problems 10th Revision. WHO, 2016 (https://icd.who.int/browse10/2016/en).

11 UK Government. Mental Health Act 1983. HMSO, 1983 (https://www. legislation.gov.uk/ukpga/1983/20/contents).

12 Felitti VJ, Anda R F, Nordenberg D, Williamson D, Spitz A, Edwards V, et al. Relationship of childhood abuse and household dysfunction to many of the leading causes of death in adults. The Adverse
Childhood Experiences (ACE) Study. Am J Prev Med 1998; 14(4): 245-58.

13 Ribeiro RB, Tully J, Fotiadou M. Clinical characteristics and outcomes on discharge of women admitted to a medium secure unit over a 4-year period. Int J Law Psychiatry 2015; 39: 83-9.

14 Baker A, Wright K, Hansen E. A qualitative study exploring female patients' experiences of self-harm in a medium secure unit. J Psychiatr Ment Health Nurs 2012; 20(9): 821-9.

15 Muskett C. Trauma-informed care in inpatient mental health settings: a review of the literature. Int J Ment Health Nurs 2014; 23(1): 51-9.

16 Cleare S, Weatherall K, Clark A, Ryan C, Kitley O, Smith M, et al. Adverse childhood experiences and hospital-treated self-harm. Int Environ Res Public Health 2018; 15(6): 1235

17 Hare Duke L, Furtado V, Guo B, Völlm B. Long-stay in forensic-psychiatric care in the UK. Soc Psychiatry Psychiatr Epidemiol 2018; 53(3): 313-21.

18 Klonsky ED, Glenn CR. Assessing the functions of non-suicidal self-injury: psychometric properties of the Inventory of Statements About Self-injury (ISAS). J Psychopathol Behav Assess 2009; 31(3): 215-9.

\title{
AGAINST THE STREAM
}

\section{Psychiatrists should investigate their patients less}

\author{
Matthew Butler, ${ }^{1,2} \odot$ Fraser Scott, ${ }^{2}$ Biba Stanton, ${ }^{2,3}$ Jonathan Rogers ${ }^{4} \odot$
}

BJPsych Bulletin (2022) 46, 152-156, doi:10.1192/bjb.2021.125

${ }^{1}$ King's College London, UK; ${ }^{2}$ South London and Maudsley NHS Foundation Trust, London, UK; ${ }^{3}$ King's College Hospital, London, UK; ${ }^{4}$ University College London, UK

Correspondence to Matthew Butler (matthew.butler@kcl.ac.uk)

First received 11 Jun 2021, final revision 1 Oct 2021, accepted 10 Nov 2021

(c) The Author(s), 2021. Published by Cambridge University Press on behalf of the Royal College of Psychiatrists. This is an Open Access article, distributed under the terms of the Creative Commons Attribution licence (https:// creativecommons.org/licenses/by/4. $0 /$ ), which permits unrestricted re-use, distribution, and reproduction in any medium, provided the original work is properly cited.
Summary Psychiatrists often order investigations such as blood tests, neuroimaging and electroencephalograms for their patients. Rationales include ruling out 'organic' causes of psychiatric presentations, providing baseline parameters before starting psychotropic medications, and screening for general cardiometabolic health. Hospital protocols often recommend an extensive panel of blood tests on admission to a psychiatric ward. In this Against the Stream article, we argue that many of these investigations are at best useless and at worst harmful: the yield of positive findings that change clinical management is extremely low; special investigations are a poor substitute for a targeted history and examination; and incidental findings may cause anxiety and further unwarranted investigation. Cognitive and cultural reasons why over-investigation continues are discussed. We conclude by encouraging a more targeted approach guided by a thorough bedside clinical assessment.

Keywords Service users; in-patient treatment; imaging; ethics; cost-effectiveness.
The Royal College of Psychiatrists' Standards for Inpatient Mental Health Services require a 'comprehensive physical review' to be initiated within four hours of admission to a psychiatric inpatient unit, which is to be completed within one week. ${ }^{1}$ In practice, this means that inpatients are often subjected to a battery of admission blood tests that are directed without regard for the presentation. Routine blood tests on admission remain a requirement in local trust policies, ${ }^{2}$ and clinical evaluations indicate that the majority of patients receive them. ${ }^{3}$ In addition, selected psychiatric patients may have further investigations, such as a magnetic resonance imaging (MRI) of the brain for those with a first psychotic episode, or an electroencephalogram (EEG) to rule out seizures. 